\title{
Convolution algebras with weighted rearrangement-invariant norm
}

\author{
by
}

R. KERMAN (St. Catharines, Ont.) and E. S A W Y ER (Hamilton, Ont.)

Abstract. Let $X$ be a rearrangement-invariant space of Lebesgue-measurable functions on $\mathbb{R}^{n}$, such as the classical Lebesgue, Lorentz or Orlicz spaces. Given a nonnegative, measurable (weight) function on $\mathbb{R}^{n}$, define $X(w)=\left\{F: \mathbb{R}^{n} \rightarrow \mathbb{C}: \infty>\|F\|_{X(w)}:=\right.$ $\left.\|F w\|_{X}\right\}$. We investigate conditions on such a weight $w$ that guarantee $X(w)$ is an algebra under the convolution product $F * G$ defined at $x \in \mathbb{R}^{n}$ by $(F * G)(x)=\int_{\mathbb{R}^{n}} F(x-y) G(y) d y$; more precisely, when $\|F * G\|_{X(w)} \leq\|F\|_{X(w)}\|G\|_{X(w)}$ for all $F, G \in X(w)$.

1. Introduction. A weight function on $\mathbb{R}^{n}$ is a Lebesgue-measurable function $w$ for which $0<w<\infty$ a.e. with respect to Lebesgue measure. Given $1 \leq p \leq \infty$, define

$$
L^{p}(w)=\left\{F: \mathbb{R}^{n} \rightarrow \mathbb{C}: \infty>\|F\|_{L^{p}(w)}=\left[\int_{\mathbb{R}^{n}}|F(x) w(x)|^{p} d x\right]^{1 / p}\right\}
$$

When $w \equiv 1$ we use the abbreviated notations $L^{p}$ and \|\|$_{p}$. As usual, $p^{\prime}=p /(p-1)$.

This paper was motivated by the problem of determining when $L^{p}(w)$ is an algebra under the convolution product $F * G$ defined at $x \in \mathbb{R}^{n}$ by

$$
(F * G)(x)=\int_{\mathbb{R}^{n}} F(x-y) G(y) d y ;
$$

more precisely, when

$$
\|F * G\|_{L^{p}(w)} \leq\|F\|_{L^{p}(w)}\|G\|_{L^{p}(w)} \quad \text { for } F, G \in L^{p}(w) .
$$

The problem was solved in the case $p=1$ by Beurling [2] who showed (1) holds if and only if

$$
w(x+y) \leq w(x) w(y) \quad \text { for } x, y \in \mathbb{R}^{n},
$$

1991 Mathematics Subject Classification: Primary 46E30, 42A85.

Research supported in part by grants from the National Science and Engineering Research Council of Canada. 
or, equivalently, setting $w(x)=e^{\Phi(x)}$,

$$
\Phi(x+y) \leq \Phi(x)+\Phi(y) \quad \text { for } x, y \in \mathbb{R}^{n} .
$$

We observe that a natural class of weights for which (2) holds is the class $\mathcal{C}$ consisting of those $w=e^{\Phi}$, where $\Phi(x)=\Phi(|x|)$ is radial and, considered as a function on $\mathbb{R}_{+}=(0, \infty), \Phi$ is increasing and concave with $\Phi(0+)=0$. Examples of such weights are $(1+|x|)^{\alpha}, \alpha \geq 0$, and $e^{|x|^{\beta}}, 0 \leq \beta \leq 1$. Here $|x|$ can be any norm on $\mathbb{R}^{n}$. However, for $n \geq 2$, the methods used below require the norm $|x|=\left|x_{1}\right|+\ldots+\left|x_{n}\right|$ for $x=\left(x_{1}, \ldots, x_{n}\right)$ in $\mathbb{R}^{n}$, which we adopt from now on. Given $x_{0} \in \mathbb{R}^{n}, r>0$, we denote by $B_{r}\left(x_{0}\right)$ the set $\left\{x \in \mathbb{R}^{n}:\left|x-x_{0}\right|<r\right\}$.

Another case readily dealt with is $p=\infty$. The weights $w$ satisfying (1) are those for which $w\left(w^{-1} * w^{-1}\right) \leq 1$; that is,

$$
\int_{\mathbb{R}^{n}} \frac{w(x)}{w(x-y) w(y)} d y \leq 1 \quad \text { for } x \in \mathbb{R}^{n} .
$$

This, together with (2) written in the form

$$
\frac{w(x)}{w(x-y) w(y)} \leq 1 \quad \text { for } x \in \mathbb{R}^{n},
$$

suggests, for $1<p<\infty$, the condition

$$
\left[\int_{\mathbb{R}^{n}}\left(\frac{w(x)}{w(x-y) w(y)}\right)^{p^{\prime}} d y\right]^{1 / p^{\prime}} \leq 1 \quad \text { for } x \in \mathbb{R}^{n} .
$$

Nikol'skiu [12] showed (4) is sufficient for (1) in the context of sequence spaces. See also Grabiner [7]. The short proof, which it will be convenient for us to reproduce here, is a clever application of Hölder's inequality. Observe first that, writing $F=f / w, G=g / w$, (1) becomes

$$
\left\|w\left(\frac{f}{w} * \frac{g}{w}\right)\right\|_{p} \leq\|f\|_{p}\|g\|_{p} .
$$

Now,

$$
\begin{aligned}
& {\left[\int_{\mathbb{R}^{n}}\left|w\left(\frac{f}{w} * \frac{g}{w}\right)\right|^{p} d x\right]^{1 / p}} \\
& \quad \leq\left[\int_{\mathbb{R}^{n}}\left[\int_{\mathbb{R}^{n}} \frac{w(x)}{w(x-y) w(y)}|f(x-y)||g(y)| d y\right]^{p} d x\right]^{1 / p}
\end{aligned}
$$




$$
\begin{aligned}
& \leq\left[\int_{\mathbb{R}^{n}}\left[\int_{\mathbb{R}^{n}}\left(\frac{w(x)}{w(x-y) w(y)}\right)^{p^{\prime}} d y\right]^{p-1}\left[\int_{\mathbb{R}^{n}}|f(x-y) g(y)|^{p} d y\right] d x\right]^{1 / p} \\
& \leq \underset{x \in \mathbb{R}^{n}}{\operatorname{ess} \sup }\left[\int_{\mathbb{R}^{n}}\left(\frac{w(x)}{w(x-y) w(y)}\right)^{p^{\prime}} d y\right]^{1 / p^{\prime}}\|\| f(x-y) g(y)\left\|_{L^{p}(d y)}\right\|_{L^{p}(d x)} .
\end{aligned}
$$

The first factor in the last line of (5) is, by (4), at most 1, while Fubini's theorem can be applied to the second factor to yield

$$
\|\| f(x-y) g(y)\left\|_{L^{p}(d y)}\right\|_{L^{p}(d x)}=\|f\|_{p}\|g\|_{p} .
$$

This proves (1).

Condition (4) is not, in general, necessary for (1). But, as we will show in Section 4 , it is if $w$ is in a certain class containing $\mathcal{C}$ (cf. [10] for a result similar to this in the case $n=1$ ).

The main purpose of this paper is to investigate when

$$
X(w)=\left\{F: \mathbb{R}^{n} \rightarrow \mathbb{C}: \infty>\|F\|_{X(w)}=\|F w\|_{X}\right\}
$$

is closed under convolution, where $X$ is a rearrangement-invariant (r.i.) space of functions on $\mathbb{R}^{n}$ with Köthe dual $X^{\prime}$. See Section 2 for definitions and some properties of such spaces. For more background we recommend [1].

Inequalities (5) with $X$ and $X^{\prime}$ in place of $L^{p}$ and $L^{p^{\prime}}$, respectively, suggest the condition

$$
\underset{x \in \mathbb{R}^{n}}{\operatorname{ess} \sup }\left\|\frac{w(x)}{w(x-y) w(y)}\right\|_{X^{\prime}(d y)} \leq C
$$

is sufficient for

$$
\|F * G\|_{X(w)} \leq C\|F\|_{X(w)}\|G\|_{X(w)},
$$

which would certainly, by Nikol'skiî's argument (5), be true if the following (weaker) analogue of (6) held:

$$
\|\| f(x-y) g(y)\left\|_{X(d y)}\right\|_{X(d x)} \leq C\|f\|_{X}\|g\|_{X} .
$$

(We would like to point out that (8) is equivalent to $X(w)$ being closed under convolution, see [8], p. 471, and that one can take $C=1$ if $w$ is replaced by $w / C$.) Now, on the one hand, (7) is no longer sufficient for (8); in particular, as shown in Section 5, (7) guarantees (8) for the Lorentz space $X=L^{p q}(\mathbb{R}), q \geq p$, if and only if $p=q$. On the other hand, as shown in Section 2, (9) does hold for nonnegative $f$ and $g$ in the class $\mathcal{R}$.D. of radially decreasing functions; that is, $f(x)=f(|x|)$ and $g(x)=g(|x|)$ are decreasing functions of $|x|$. This, then, raises the question of characterizing those weights for which it is enough to test (8) for nonnegative functions in R.D. 
To this end, we introduce the class $\mathcal{M}$ of weights $w(x)=w(|x|)$ for which $w(y) \leq C w(z), 0<y<z$, and

$$
B(r, s)=\frac{w(r+s)}{w(r) w(s)} \quad(r, s>0)
$$

is essentially decreasing in each variable separately, i.e. $B\left(r_{1}, s\right) \leq C B\left(r_{2}, s\right)$, whenever $s>0$ and $r_{1} \geq r_{2}>0$. (This class contains $\mathcal{C}$, since for $w=e^{\Phi}$, $\Phi$ concave on $\mathbb{R}_{+}, \partial B / \partial r=\left(\Phi^{\prime}(r+s)-\Phi^{\prime}(r)\right) B(r, s) \leq 0$.) We prove that given $w \in \mathcal{M}$ there holds the following weighted analogue of an inequality of F. Riesz [13] and S. L. Sobolev [14]:

$$
\int_{\mathbb{R}^{n}}\left(\frac{f}{w} * \frac{g}{w}\right) h w \leq C \int_{\mathbb{R}^{n}}\left(\frac{f^{+}}{w} * \frac{g^{+}}{w}\right) h^{++} w \quad \text { for } f, g, h \geq 0 .
$$

Here, for example, $h^{+}$is the (a.e.) unique nonnegative function in $\mathcal{R}$.D. on $\mathbb{R}^{n}$ satisfying

$$
\left|\left\{x \in \mathbb{R}^{n}: h^{+}(|x|)>\lambda\right\}\right|=\left|\left\{x \in \mathbb{R}^{n}:|h(x)|>\lambda\right\}\right|
$$

for all $\lambda>0$, and $h^{++}$is the (larger) nonnegative $\mathcal{R}$.D. function on $\mathbb{R}^{n}$ given by

$$
h^{++}(x)=h^{++}(|x|)=\left(C_{n}|x|\right)^{-n} \int_{|y| \leq|x|} h^{+}(|y|) d y,
$$

where $C_{n}^{n}=\left|B_{1}(0)\right|$.

In sum, we are able to prove the following

THEOREM 1. Let $w \in \mathcal{M}$ and suppose $X$ is an r.i. space of functions on $\mathbb{R}^{n}$ for which the mapping $f \rightarrow f^{++}$is bounded on $X^{\prime}$. Then a necessary and sufficient condition for $X(w)$ to be closed under convolution is

$$
\left\|\frac{w(x)}{w(x-y) w(y)}\right\|_{X^{\prime}(d y)} \leq C \quad \text { for } x \in \mathbb{R}^{n} .
$$

The requirement that $f \rightarrow f^{++}$be bounded on $X^{\prime}$ eliminates those r.i. spaces $X$ near $L^{\infty}$ (see Lemma 6 below). To include such spaces in our theory requires a stronger weighted analogue of the Riesz inequality, namely (10) with $h^{+}$in place of $h^{++}$; that is,

$$
\int_{\mathbb{R}^{n}}\left(\frac{f}{w} * \frac{g}{w}\right) h w \leq C \int_{\mathbb{R}^{n}}\left(\frac{f^{+}}{w} * \frac{g^{+}}{w}\right) h^{+} w \quad \text { for } f, g, h \geq 0 .
$$

We show that for $w \in \mathcal{M}_{\infty}$, where

$$
\mathcal{M}_{\infty}=\left\{w \in \mathcal{M}: \frac{w(x+y)}{w(x) w(y)} \in L^{\infty}\left(\mathbb{R}^{n} \times \mathbb{R}^{n}\right)\right\},
$$


(12) holds if and only if $w$ satisfies the additional condition

$$
\int_{0}^{r / 2} \frac{w(r)}{w(r-s) w(s)} s^{n-1} d s \leq C \int_{0}^{r} \frac{s^{n-1}}{w(s)^{2}} d s \quad \text { for } r>0 .
$$

(We note in passing that $w(x)=e^{|x|^{\alpha}}, x \in \mathbb{R}^{n}$, belongs to $\mathcal{C} \subset \mathcal{M}_{\infty}$ for $0 \leq \alpha \leq 1$, but satisfies (13) if and only if $\alpha=1$. Indeed, if $\alpha=1$ the left side is $\approx r^{n}$, while the right side is $O(1)$.) We can now obtain the following result having no restriction on $X$.

Theorem 2. Let $w(x)=w(|x|)$ belong to $\mathcal{M}_{\infty}$ and satisfy (13). Suppose $X$ is an r.i. space of functions on $\mathbb{R}^{n}$. Then (11) is a necessary and sufficient condition for $X(w)$ to be closed under convolution.

The sufficiency of (11) is related to (10) and (12) in Section 2 and proofs of the latter are given in the following section. The necessity of (11) is the subject of Section 4 and, as mentioned above, the question of the general sufficiency of (11) is considered for the $L^{p q}$ spaces, $q \geq p$ (when $n=1$ ), in Section 5.

The referee has pointed out that it should be possible to extend some of our results to the setting of locally compact Abelian groups.

2. Rearrangement-invariant function spaces. Let $(\Omega, \Sigma, \mu)$ be a complete $\sigma$-finite measure space. A Banach lattice $X=X(\Omega)$ is a Banach space of (equivalence classes of $\mu$-a.e. equal) complex-valued measurable functions on $\Omega$ such that if $|g| \leq|f| \mu$-a.e., where $f \in X$ and $g$ is measurable, then $g \in X$ and $\|g\|_{X} \leq\|f\|_{X}$. If, in addition, $X$ has the Fatou property:

$$
0 \leq f_{n} \uparrow f \mu \text {-a.e., } \sup _{n}\left\|f_{n}\right\|_{X}<\infty \Rightarrow f \in X \text { and }\|f\|_{X}=\lim _{n \rightarrow \infty}\left\|f_{n}\right\|_{X}
$$

together with the property that whenever $E \in \Sigma$ with $\mu(E)<\infty$ we have $\chi_{E} \in X$ and $\int_{\Omega}|f| \chi_{E} d \mu<\infty$ for all $f \in X$, then $X$ is said to be a Banach function space. Such a space is a saturated Banach lattice in the sense that every $E \in \Sigma$ with $\mu(E)>0$ has a measurable subset $F$ of finite positive measure for which $\chi_{F} \in X$.

The Banach function space $X=X(\Omega)$ is called a rearrangement-invariant function space (r.i. space) if $f \in X$ implies $g \in X$ and $\|g\|_{X}=\|f\|_{X}$, whenever $g$ is equimeasurable with $f$, that is,

$$
\begin{aligned}
\mu_{f}(t) & :=\mu(\{x \in \Omega:|f(x)|>t\}) \\
& =\mu(\{x \in \Omega:|g(x)|>t\})=: \mu_{g}(t) \quad \text { for } t>0 .
\end{aligned}
$$

Important examples of r.i. spaces are the Lorentz spaces $L^{p q}(\Omega), 1<p<\infty$, 
$1 \leq q \leq \infty$, with norms given by

$$
\|f\|_{p q}= \begin{cases}\left\{\int_{0}^{\infty}\left(s \mu_{f}(s)^{1 / p}\right)^{q} s^{-1} d s\right\}^{1 / q} & \text { for } q<\infty \\ \sup _{s>0} s \mu_{f}(s)^{1 / p} & \text { for } q=\infty .\end{cases}
$$

In case $p=q, L^{p q}(\Omega)=L^{p}(\Omega)$, the usual Lebesgue space, and we shorten $\|f\|_{p p}$ to $\|f\|_{p}$. The smallest of all r.i. spaces is the intersection, $L^{1} \cap L^{\infty}$, of $L^{1}(\Omega)$ and $L^{\infty}(\Omega)$, with $\|f\|_{L^{1} \cap L^{\infty}}=\max \left\{\|f\|_{1},\|f\|_{\infty}\right\}$.

The Köthe dual or associate space $X^{\prime}=X^{\prime}(\Omega)$ of a Banach lattice $X=X(\Omega)$ consists of those complex-valued measurable functions $f$ on $\Omega$ such that $f g \in L^{1}(\Omega)$ for all $g \in X$. We define

$$
\|f\|_{X^{\prime}}=\sup \left\{\left|\int_{\Omega} f g d \mu\right|:\|g\|_{X} \leq 1\right\} .
$$

This is a norm provided $X$ has the Fatou property. In this case, $X^{\prime}$ is a Banach lattice which is both saturated and has the Fatou property; moreover, $X^{\prime \prime}=X$ isometrically, so that

$$
\|f\|_{X}=\sup \left\{\left|\int_{\Omega} f g d \mu\right|:\|g\|_{X^{\prime}} \leq 1\right\} .
$$

The generalized Hölder inequality asserts the consequence of (14) that when $f \in X, g \in X^{\prime}$, the function $f g \in L^{1}(\Omega)$ and $\|f g\|_{1} \leq\|f\|_{X}\|g\|_{X^{\prime}}$. Theorem 5.2 of [1] shows that for $X$ an r.i. space on $\mathbb{R}^{n}$,

$$
\left\|\chi_{B_{r}(0)}\right\|\left\|_{X}\right\| \chi_{B_{r}(0)} \|_{X^{\prime}}=C_{n} r^{n} \quad \text { for } r>0 .
$$

Given Banach lattices $X=X(\Omega), Y=Y(\Omega)$ and $0<\theta<1$, the Calderón product $Z=X^{1-\theta} Y^{\theta}$ consists of all measurable $h$ on $\Omega$ such that $|h| \leq \lambda f^{1-\theta} g^{\theta} \mu$-a.e. for some $\lambda>0,0 \leq f \in X, 0 \leq g \in Y,\|f\|_{X}$, $\|g\|_{Y} \leq 1$. In this case, $\|h\|_{Z}=\inf \lambda$. It is shown in \#33.5 of [4] that $Z$ is a Banach lattice. Further, one readily proves that $Z$ is saturated whenever $X$ and $Y$ are and that it has the Fatou property whenever $X$ and $Y$ do.

Theorem 3. Let $X_{i}=X_{i}(\Omega), Y_{i}=Y_{i}(\Omega), i=1,2$, be Banach lattices which have the Fatou property and let $X_{\theta}=X_{1}^{1-\theta} X_{2}^{\theta}, Y_{\theta}=Y_{1}^{1-\theta} Y_{2}^{\theta}$ for some fixed $\theta, 0<\theta<1$. Suppose $T$ is a linear operator which satisfies

$$
0 \leq g_{n} \uparrow g \in X_{i} \mu \text {-a.e. } \Rightarrow 0 \leq T g_{n} \uparrow T g \in Y_{i} \mu \text {-a.e. }
$$

with

$$
\left\|T f_{i}\right\|_{Y_{i}} \leq M_{i}\left\|f_{i}\right\|_{X_{i}} \quad \text { for } f_{i} \in X_{i}, i=1,2
$$

Then

$$
\|T f\|_{Y_{\theta}} \leq M_{\theta}\|f\|_{X_{\theta}} \quad \text { for } f \in X_{\theta} \text {, where } M_{\theta} \leq M_{1}^{1-\theta} M_{2}^{\theta} .
$$

Proof. Consider $f \in X_{\theta}$ with

$$
|f| \leq \lambda g^{1-\theta} h^{\theta} \mu \text {-a.e., } \lambda>0 ; \quad g, h \geq 0 ; \quad\|g\|_{X_{1}},\|h\|_{X_{2}} \leq 1 .
$$


Then, by the abstract Hölder inequality ([9], p. 143)

$$
\begin{aligned}
|T f| & \leq T|f| \leq \lambda T\left(g^{1-\theta} h^{\theta}\right) \leq \lambda[T g]^{1-\theta}[T h]^{\theta} \\
& \leq \lambda M_{1}^{1-\theta} M_{2}^{\theta}\left(\frac{T g}{M_{1}}\right)^{1-\theta}\left(\frac{T h}{M_{2}}\right)^{\theta} .
\end{aligned}
$$

Hence, $\|T h\|_{Y_{\theta}} \leq \lambda M_{1}^{1-\theta} M_{2}^{\theta}$ and we are done.

TheOrem 4 (Lozanovskiu [11]). Let $X=X(\Omega)$ be a Banach lattice with Köthe dual $X^{\prime}=X^{\prime}(\Omega)$. Suppose $X$ (and hence $\left.X^{\prime}\right)$ is saturated and has the Fatou property. Set $Z=X(\Omega)^{1 / 2} X^{\prime}(\Omega)^{1 / 2}$. Then $Z=L^{2}(\Omega)$ isometrically.

Proof. We begin by observing that $Z$ is a saturated Banach lattice which has the Fatou property.

Given $f \in Z$, let $\lambda>0$ be such that $|f| \leq \lambda g^{1 / 2} h^{1 / 2}$ for $0 \leq g \in X$, $0 \leq h \in X^{\prime}$, with $\|g\|_{X},\|h\|_{X^{\prime}} \leq 1$. Then

$$
\|f\|_{2}=\left(\int_{\Omega}|f|^{2} d \mu\right)^{1 / 2} \leq \lambda\left(\int_{\Omega} g h d \mu\right)^{1 / 2} \leq \lambda\left(\|g\|_{X}\|h\|_{X^{\prime}}\right)^{1 / 2} \leq \lambda
$$

and so $\|f\|_{2} \leq\|f\|_{Z}$. Here, we have used Hölder's inequality.

Suppose, next, that $f \in L^{2}(\Omega)$ and $\|f\|_{2}=1$. We have $|f|=\sqrt{|f|^{2}}$, where $|f|^{2} \in L^{1}(\Omega)$ and $\left\||f|^{2}\right\|_{1}=1$. By Theorem 1 in $[6],|f|^{2}=g h$, where $\|g\|_{X}\|h\|_{X^{\prime}}=1$; indeed, without loss of generality, $\|g\|_{X}=\|h\|_{X^{\prime}}=1$. It follows that $\|f\|_{Z} \leq 1=\|f\|_{2}$. The same is then clearly true of any $f \in L^{2}(\Omega)$. This completes the proof.

Given a Banach lattice $X=X(\Omega)$ and measurable $w: \Omega \rightarrow \mathbb{R}_{+}$, define

$$
X(w)=\left\{F: \Omega \rightarrow \mathbb{C}: \infty>\|F\|_{X(w)}=\|F w\|_{X}\right\} .
$$

It is easily seen $X(w)$ is a Banach lattice which is saturated whenever $X$ is and has the Fatou property whenever $X$ does; further, $X(w)^{\prime}=X^{\prime}\left(w^{-1}\right)$. We thus have

Corollary 5. Let $X=X(\Omega)$ be a Banach lattice with Köthe dual $X^{\prime}=X^{\prime}(\Omega)$ and assume $X$ (and hence $X^{\prime}$ ) is saturated and has the Fatou property. Suppose $w: \Omega \rightarrow \mathbb{R}_{+}$is measurable. Then $X(w)^{1 / 2} X^{\prime}\left(w^{-1}\right)^{1 / 2}=$ $L^{2}(\Omega)$ isometrically.

We now record two additional results for r.i. spaces, the first of which characterizes one of the hypotheses in Theorem 1.

Lemma 6 (D. Boyd [3]). Suppose $X=X\left(\mathbb{R}^{n}\right)$ is an r.i. space of functions on $\mathbb{R}^{n}$. Then the mapping $f \rightarrow f^{++}$is bounded on $X$ if and only if $\lim _{s \rightarrow \infty} h(s)=0$, where $h(s)$ is the (finite) operator norm of the dilation operator $\left(E_{s} f\right)(x):=f(s x)\left(s>0, x \in \mathbb{R}^{n}\right)$ from $X$ to itself. 
Lemma 7. Suppose $X=X\left(\mathbb{R}^{n}\right)$ is an r.i. space of functions on $\mathbb{R}^{n}$. Then there is a positive constant $C$ such that for all $0 \leq f, g \in \mathcal{R}$.D.,

$$
\|\| f(x-y) g(y)\left\|_{X(d y)}\right\|_{X(d x)} \leq C\|f\|_{X}\|g\|_{X} .
$$

Proof. Given $0 \leq f, g \in \mathcal{R}$.D. and $x, y \in \mathbb{R}^{n}$, we have

$$
f(x-y) g(y)=f(|x-y|) g(|y|) \leq f(|x| / 2) g(|y|)+f(|x-y|) g(|x| / 2),
$$

since $f(|x-y|) \leq f(|x| / 2)$ if $|x-y| \geq|x| / 2$ while $g(|y|) \leq g(|x| / 2)$ if $|y| \geq|x| / 2$ (one of these cases must hold as $|x| \leq|x-y|+|y|$ ). Thus, the left side of (16) is at most

$$
\begin{aligned}
& \|\| f(|x| / 2) g(|y|)+f(|x-y|) g(|x| / 2)\left\|_{X(d y)}\right\|_{X(d x)} \\
& \quad \leq\|f(|x| / 2)\|_{X}\|g\|_{X}+\|f\|_{X}\|g(|x| / 2)\|_{X} \leq 2 h(1 / 2)\|f\|_{X}\|g\|_{X},
\end{aligned}
$$

where we have used the fact that $X$ is translation-invariant.

Finally, we show how the sufficiency of (11) for $X(w)$ to be closed under convolution reduces to (10) (in Theorem 1) and (12) (in Theorem 2). Indeed, assuming first the hypotheses of Theorem 1 we have, by (15), with $F=$ $f / w \geq 0, G=g / w \geq 0$,

$$
\begin{aligned}
& \|F * G\|_{X(w)}=\sup _{\substack{\|h\|_{X^{\prime} \leq 1} \leq 1 \\
h \geq 0}} \int_{\mathbb{R}^{n}}\left(\frac{f}{w} * \frac{g}{w}\right) h w \\
& \leq \sup _{\substack{\|h\|_{X^{\prime} \leq 1} \leq 1 \\
h \geq 0}} \int_{\mathbb{R}^{n}}\left(\frac{f^{+}}{w} * \frac{g^{+}}{w}\right) h^{++} w \quad \text { given }(10) \\
& \leq C \sup _{\substack{\|h\|_{X^{\prime} \leq 1} \leq 1 \\
h \geq 0}}\left\|\left(\frac{f^{+}}{w} * \frac{g^{+}}{w}\right) w\right\|_{X}\left\|h^{++}\right\|_{X^{\prime}} \quad \text { by Hölder's inequality } \\
& \leq C\left\|\left(\frac{f^{+}}{w} * \frac{g^{+}}{w}\right) w\right\|_{X} \quad \text { since } h \rightarrow h^{++} \text {is bounded on } X^{\prime} \\
& \leq C \operatorname{ess}_{x \in \mathbb{R}^{n}}\left\|\frac{w(x)}{w(x-y) w(y)}\right\|_{X^{\prime}(d y)}\|\| f^{+}(x-y) g^{+}(y)\left\|_{X(d y)}\right\|_{X(d x)} \\
& \text { as in (5) } \\
& \leq C\left\|f^{+}\right\|_{X}\left\|g^{+}\right\|_{X}=C\|f\|_{X}\|g\|_{X}=\|F\|_{X}(w)\|G\|_{X}(w),
\end{aligned}
$$

since, by Lemma 7, (9) holds for the nonnegative $\mathcal{R}$.D . functions $f^{+}, g^{+}$.

Assuming the hypotheses of Theorem 2 instead, we again obtain (17), but this time with $\left\|h^{+}\right\|_{X^{\prime}}=\|h\|_{X^{\prime}}$ in place of $\left\|h^{++}\right\|_{X^{\prime}}$ (by (12)) and since $\|h\|_{X^{\prime}} \leq 1$, no assumption on $X^{\prime}$ is needed now. 
3. The weighted Riesz-Sobolev inequalities. As shown in the last section, the sufficiency of (11) for $X(w)$ to be closed under convolution depends, under varying assumptions on $X$ and $w$, on the following two theorems:

Theorem 8. Suppose $w \in \mathcal{M}$. Then

(18) $\int_{\mathbb{R}^{n}}\left(\frac{f}{w} * \frac{g}{w}\right) h w \leq C \int_{\mathbb{R}^{n}}\left(\frac{f^{+}}{w} * \frac{g^{+}}{w}\right) h^{++} w \quad$ for $f, g, h \geq 0$.

Theorem 9. Suppose $w \in \mathcal{M}_{\infty}$. Then

$$
\int_{\mathbb{R}^{n}}\left(\frac{f}{w} * \frac{g}{w}\right) h w \leq C \int_{\mathbb{R}^{n}}\left(\frac{f^{+}}{w} * \frac{g^{+}}{w}\right) h^{+} w \quad \text { for } f, g, h \geq 0
$$

if and only if $w$ satisfies (13).

The proofs of Theorems 8 and 9 require certain monotonicity properties of $w \in \mathcal{M}$. These are a consequence of the following general result.

Lemma 10. Suppose $\Phi: \mathbb{R}^{n} \rightarrow \mathbb{R}^{n}$ satisfies $\Phi(x)=\Phi(|x|), x \in \mathbb{R}^{n}$. If there exists $C>0$ such that

$$
\Phi(x) \leq C \Phi(z) \quad \text { for }|x| \geq|z|
$$

then

$$
\int_{|x| \geq r} \chi_{E}(x) \Phi(x) d x \leq C|E| \min \left\{\Phi(r),|F|^{-1} \int_{F} \Phi(y) d y\right\}
$$

for all $E \subset \mathbb{R}^{n}, r>0$ and $F \subset B_{r}(0)$. In particular,

(22) $\int_{E} \Phi(x) d x \leq\left(C^{2}+1\right) \int_{|x| \leq r_{n}} \Phi(x) d x \quad$ for $E \subset \mathbb{R}^{n}, r_{n}=C_{n}^{-1}|E|^{1 / n}$.

If there exists $C>0$ such that

$$
\Phi(x) \leq C\left(C_{n}|x|\right)^{-n} \int_{|y| \leq|x|} \Phi(y) d y \quad \text { for } x \in \mathbb{R}^{n},
$$

then

$$
\int_{|x| \leq r} \chi_{E}(x) \Phi(x) d x \leq(C+1) \int_{|x| \leq r} \chi_{E}^{++}(x) \Phi(x) d x,
$$

for all $E \subset \mathbb{R}^{n}$ and $r>0$.

P r o o f. We obtain (21) from (20) since $\Phi(x) \leq C \Phi(r)$ and $\Phi(x) \leq C \Phi(y)$ whenever $|x|>r$ and $|y|<r$. Then (22) follows on writing

$$
\int_{E} \Phi(x) d x=\int_{|x|<r_{n}} \chi_{E}(x) \Phi(x) d x+\int_{|x| \geq r_{n}} \chi_{E}(x) \Phi(x) d x
$$

and applying (21) with $F=B_{r_{n}}(0)$. 
If $r \leq r_{n}$, then (24) is trivial, so we suppose $r>r_{n}$. We have

$$
\begin{aligned}
\int_{|x| \leq r} \chi_{E}(x) & \Phi(x) d x \\
& \leq \int_{|x| \leq r_{n}} \Phi(x) d x+C \int_{r_{n} \leq|x| \leq r} \chi_{E}(x)\left\{\left(C_{n}|x|\right)^{-n} \int_{|z| \leq|x|} \Phi(z) d z\right\} d x \\
& \leq \int_{|x| \leq r_{n}} \Phi(x) d x+C \int_{|z| \leq r} \Phi(z)\left\{\int_{\max \left(|z|, r_{n}\right)}^{r} \chi_{E}(x)\left(C_{n}|x|\right)^{-n} d x\right\} d z \\
& \leq \int_{|x| \leq r_{n}} \Phi(x) d x+C \int_{|z| \leq r} \Phi(z) \frac{|E|}{\max \left\{\left(C_{n}|x|\right)^{n},|E|\right\}} d z \\
& \leq(C+1) \int_{|x| \leq r} \chi_{E}^{++}(x) \Phi(x) d x .
\end{aligned}
$$

Lemma 11. Suppose $w \in \mathcal{M}$. Then

(i) $B(|x|, \cdot)$ and $B(\cdot,|x|)$ satisfy (20) with $C>0$ independent of $x \in \mathbb{R}^{n}$;

(ii) $W(x)=\int_{0}^{|x|} \frac{w(|x|)}{w(|x|-s) w(s)} s^{n-1} d s$ satisfies (23).

Proof. (i) is obvious. To prove (ii) we first show that if $3|x| / 4 \leq|y| \leq$ $|x|$, then $W(x) \leq C W(y)$. Now,

$$
W(x)=\left(\int_{0}^{|x| / 2}+\int_{|x| / 2}^{|x|}\right) \frac{w(|x|)}{w(|x|-s) w(s)} s^{n-1} d s=I+I I .
$$

Since $w \in \mathcal{M}$, we have

$$
I \leq \int_{0}^{|x| / 2} \frac{w(|y|)}{w(|y|-s) w(s)} s^{n-1} d s \leq W(|y|),
$$

and

$$
\begin{aligned}
I I & \leq \int_{|x| / 2}^{|x|} \frac{w(|y|)}{w(|x|-s) w(s+|y|-|x|)} s^{n-1} d s \\
& \leq \int_{|y|-|x| / 2}^{|y|} \frac{w(|y|)}{w(|y|-t) w(t)}(t+|x|-|y|)^{n-1} d t \leq C_{n} W(|y|),
\end{aligned}
$$

since $t+|x|-|y| \leq C t$ for $|y|-|x| / 2 \leq t|y|$ and $3|x| / 4 \leq|y| \leq|x|$. From (25) and (26) we obtain $W(|x|) \leq C_{n} W(|y|)$ for $3|x| / 4 \leq|y| \leq|x|$, as claimed. 
Iterating this inequality yields the doubling condition

$$
W(x) \leq C\left(\frac{|x|}{|y|}\right)^{\beta} W(|y|) \quad \text { for } 0<|y| \leq|x|,
$$

where $C$ and $\beta$ are positive constants depending only on the dimension $n$. We now obtain that $W$ satisfies (23) easily from

$$
\begin{aligned}
W(x) & =C_{\beta}\left(C_{n}|x|\right)^{-n} \int_{|y| \leq|x|} W(x) \frac{|y|^{\beta}}{|x|^{\beta}} d y \\
& \leq C_{\beta}\left(C_{n}|x|\right)^{-n} \int_{|y| \leq|x|} W(|y|) d y .
\end{aligned}
$$

Let $\mathbb{R}_{+}^{n}=\left\{\left(x_{1}, \ldots, x_{n}\right): x_{1} \geq 0, \ldots, x_{n} \geq 0\right\}$. Define the set $E^{+}$by $\chi_{E^{+}}=\left(\chi_{E}\right)^{+}$; this will be a ball (recall $\left.|x|=\left|x_{1}\right|+\ldots+\left|x_{n}\right|\right)$ centred at the origin with, say, radius $r_{E^{+}}$. Lastly, denote by $\widetilde{E}$ the ball concentric with $E^{+}$and with radius $r_{\widetilde{E}}=\frac{1}{2} r_{E^{+}}$.

Proof of Theorems 8 and 9. To begin, observe that it is enough to prove (18) and (19) for nonnegative simple functions $f, g$ and $h$ which are symmetric with respect to all $2^{n}$-orthotants of $\mathbb{R}^{n}$. Furthermore, we claim that one need only consider $f=\chi_{E}, g=\chi_{F}, h=\chi_{G}$, where $E$, $F$ and $G$ are sets symmetric with respect to all $2^{n}$-orthotants of $\mathbb{R}^{n}$. For, suppose the latter fact to be true. Then the simple functions $f, g$ and $h$ referred to above can be written as finite sums of the form $f=\sum_{i} f_{i} \chi_{E_{i}}$, $g=\sum_{j} g_{j} \chi_{F_{j}}, h=\sum_{k} h_{k} \chi_{G_{k}}$, where the sets $E_{i}, F_{j}$ and $G_{k}$ are symmetric, with $E_{i} \supset E_{i+1}, F_{j} \supset F_{j+1}, G_{k} \supset G_{k+1}$ and the constants $f_{i}, g_{j}$ and $h_{k}$ are nonnegative. Hence, we get (for example) (18) as follows:

$$
\begin{aligned}
& \int_{\mathbb{R}^{n}}\left(\frac{f}{w} * \frac{g}{w}\right) h w=\sum_{i, j, k} f_{i} g_{j} h_{k} \int_{\mathbb{R}^{n}}\left(\frac{\chi_{E_{i}}}{w} * \frac{\chi_{F_{j}}}{w}\right) \chi_{G_{k}} w \\
& \quad \leq C \sum_{i, j, k} f_{i} g_{j} h_{k} \int_{\mathbb{R}^{n}}\left(\frac{\chi_{E_{i}^{+}}}{w} * \frac{\chi_{F_{j}^{+}}}{w}\right) \chi_{G_{k}}^{++} w=C \int_{\mathbb{R}^{n}}\left(\frac{f^{+}}{w} * \frac{g^{+}}{w}\right) h^{++} w .
\end{aligned}
$$

Summarizing, we have shown that in order to prove (18) and (19), it is enough to establish, respectively,

$$
\begin{aligned}
& \iint_{E \times F} \chi_{G}(x+y) \frac{w(x+y)}{w(x) w(y)} d x d y \\
& \leq C \iint_{E^{+} \times F^{+}} \chi_{G}^{++}(x+y) \frac{w(x+y)}{w(x) w(y)} d x d y,
\end{aligned}
$$


for symmetric sets $E, F, G \subset \mathbb{R}^{n}$, and

$$
\begin{aligned}
& \iint_{E \times F} \chi_{G}(x+y) \frac{w(x+y)}{w(x) w(y)} d x d y \\
& \leq C \iiint_{E^{+} \times F^{+}} \chi_{G}^{+}(x+y) \frac{w(x+y)}{w(x) w(y)} d x d y
\end{aligned}
$$

for symmetric sets $E, F, G \subset \mathbb{R}^{n}$.

To prove (27) and (28) we distinguish three cases, in all of which it may be assumed without loss of generality that $|E| \leq|F|$.

Case 1: $|E| \leq|F| \leq|G|$. In this case we actually have the stronger inequality (28) for $w \in \mathcal{M}$ without any additional assumptions. Indeed, since $E$ and $F$ are symmetric and $w(x+y) \leq C w(|x|+|y|)$, the left side of (28) is at most

$$
C \int_{F} \int_{E} B(|x|,|y|) d x d y \leq C \int_{F} \int_{\widetilde{E}} B(|x|,|y|) d x d y
$$

by Lemma 11(i) and (21) of Lemma 10, and thus at most

$$
\begin{aligned}
& \leq C \int_{\widetilde{F}} \int_{\widetilde{E}} B(|x|,|y|) d x d y \\
& =2^{n} C \underset{\mathbb{R}_{+}^{n} \times \mathbb{R}_{+}^{n}}{\iint_{\widetilde{E}}} \chi_{\widetilde{E}}(x) \chi_{\widetilde{E}}(y) B(|x|,|y|) d x d y
\end{aligned}
$$

upon reversing the order of integration and applying (21) again. Since $\widetilde{E}+$ $\widetilde{F} \subset G^{+}$and $|x+y|=|x|+|y|$ for $x, y \in \mathbb{R}_{+}^{n}$, the last integral in (29) is at most

$$
\begin{aligned}
C \iint_{\mathbb{R}_{+}^{n} \times \mathbb{R}_{+}^{n}} \chi_{E}^{+}(x) \chi_{F}^{+}(y) \chi_{G}^{+}(x+y) \frac{w(x+y)}{w(x) w(y)} & d x d y \\
& \leq C \int_{\mathbb{R}^{n}}\left(\frac{\chi_{E}^{+}}{w} * \frac{\chi_{F}^{+}}{w}\right) \chi_{G}^{+} w .
\end{aligned}
$$

C a s e $2:|E| \leq|G| \leq|F|$. Here again there holds the stronger inequality (28) assuming only $w \in \mathcal{M}$. We have

$$
\begin{aligned}
\int_{\mathbb{R}^{n}}\left(\frac{\chi_{E}}{w} * \frac{\chi_{F}}{w}\right) \chi_{G} w & \leq \iint_{E \times \mathbb{R}^{n}} \chi_{G}(x+y) B(|x|,|y|) d x d y \\
& \leq C \int_{E \cap \mathbb{R}_{+}^{n} \times \mathbb{R}_{+}^{n}} \chi_{\mathcal{G}}(x, y) B(|x|,|y|) d y d x,
\end{aligned}
$$


where $\chi_{\mathcal{G}}(x, y)=\sum \chi_{G}\left(x_{1} \pm y_{1}, \ldots, x_{n} \pm y_{n}\right)$ for $x, y \in \mathbb{R}_{+}^{n}$, the sum being extended over all choices of \pm . The last term in (30) equals

$$
C \int_{E \cap \mathbb{R}_{+}^{n}} \int_{\mathcal{G}_{x}} B(|x|,|y|) d y d x \quad \text { where } \chi_{\mathcal{G}_{x}}(y)=\chi_{\mathcal{G}}(x, y), x, y \in \mathbb{R}_{+}^{n} .
$$

Arguing as in case 1 and observing that $\left|\mathcal{G}_{x}\right| \leq 2^{n}|G|$ for all $x \in \mathbb{R}_{+}^{n}$, we obtain the upper bound

$$
\begin{aligned}
& C \int_{\mathbb{R}_{+}^{n} \times \mathbb{R}_{+}^{n}} \chi_{\widetilde{E}}(x) \chi_{\widetilde{G}}(y) \frac{w(x+y)}{w(x) w(y)} d x d y \\
& \quad \leq C \int_{\mathbb{R}_{+}^{n} \times \mathbb{R}_{+}^{n}} \chi_{\widetilde{E}}(x) \chi_{\widetilde{G}}(y) \chi_{G^{+}}(x+y) \frac{w(x+y)}{w(x) w(y)} d x d y \quad \text { since } \widetilde{E}+\widetilde{G} \subset G^{+} \\
& \quad \leq C \int_{\mathbb{R}^{n}}\left(\frac{\chi_{E}^{+}}{w} * \frac{\chi_{F}^{+}}{w}\right) \chi_{G}^{+} w .
\end{aligned}
$$

Case 3: $|G| \leq|E| \leq|F|$. In this case we can only obtain (27) for $w \in \mathcal{M}$. We then prove (28) holds for $w \in \mathcal{M}_{\infty}$ if and only if (13) does. The left side of $(27)$ is at most

$$
\left(\int_{\widetilde{E} \times \widetilde{E}}+\underset{(\widetilde{E} \times \widetilde{E})^{\mathrm{c}}}{\int} \chi_{\widetilde{E}} \chi_{G}(x+y) \frac{w(x+y)}{w(x) w(y)} d x d y=I+I I .\right.
$$

Let $r_{k}=x_{k}+y_{k}, s_{k}=y_{k}, r=\sum_{k}\left|r_{k}\right|$ and $s=\sum_{k}\left|s_{k}\right|$. Since $|r-s| \leq$ $\sum_{k}\left|r_{k}-s_{k}\right|=|x|$, we have $w(|r-s|) \leq C w(|x|)$, and we may bound $I$ by

$$
\int_{E^{+}} \chi_{G}\left(r_{1}, \ldots, r_{n}\right) \int_{0}^{r_{\tilde{E}}} \frac{w(r)}{w(|r-s|) w(s)} s^{n-1} d s d r_{1} \ldots d r_{n} .
$$

We now show that the inner integral in (32) satisfies

$$
\int_{0}^{r_{\tilde{E}}} \frac{w(r)}{w(|r-s|) w(s)} s^{n-1} d s \leq C\left[W(r)+W\left(r_{\widetilde{E}}\right)\right] .
$$

Indeed, when $r_{\widetilde{E}} \leq r \leq r_{E^{+}}$, the left side of (33) is at most $C W\left(r_{\widetilde{E}}\right)$ since $w \in \mathcal{M}$; while, for $0 \leq r \leq r_{\widetilde{E}}$, we have, letting $r_{0}=\min \left\{r, r_{\widetilde{E}}-r\right\}$ and observing that $w(r) / w(r+s) \leq C$ and $w\left(r_{\widetilde{E}}\right) / w\left(r_{\widetilde{E}}-s\right) \geq c$,

$$
\int_{0}^{r_{\tilde{E}}} \frac{w(r)}{w(|r-s|) w(s)} s^{n-1} d s=W(r)+\int_{0}^{r_{\tilde{E}}-r} \frac{w(r)}{w(r+s) w(s)}(r+s)^{n-1} d s
$$


and

$$
\begin{aligned}
& \int_{0}^{r_{\widetilde{E}}-r} \frac{w(r)}{w(r+s) w(s)}(r+s)^{n-1} d s \\
& \leq C\left[r^{n-1} \int_{0}^{r_{0}} B\left(r_{\widetilde{E}}-s, s\right) d s+\int_{r}^{r_{\tilde{E}}} B\left(r_{\widetilde{E}}-s, s\right) s^{n-1} d s\right] \\
& \leq C \int_{0}^{r_{\tilde{E}}} B\left(r_{\widetilde{E}}-s, s\right) s^{n-1} d s=C W\left(r_{\widetilde{E}}\right) .
\end{aligned}
$$

Thus,

$$
I \leq C \int_{E^{+}} \chi_{G}\left(r_{1}, \ldots, r_{n}\right) W(r) d r_{1} \ldots d r_{n}+C|G| W\left(r_{\widetilde{E}}\right) .
$$

Both terms on the right side of (34) are no larger than

$$
C \int_{E^{+}} \chi_{G}^{++}(r) W(r) d r_{1} \ldots d r_{n}
$$

this is true for the first term by Lemma 11(ii) and (24) of Lemma 10, while for the second term we have, by Lemma 11(ii) again,

$$
|G| W\left(r_{\widetilde{E}}\right) \leq C \frac{|G|}{\left(r_{\widetilde{E}}\right)^{n}} \int_{\widetilde{E}} W(r) d r_{1} \ldots d r_{n} \leq C \int_{E^{+}} \chi_{G}^{++}(r) W(r) d r_{1} \ldots d r_{n} .
$$

Since

$$
\begin{aligned}
\iint_{E \times F} \chi_{G}^{++}(x+y) & \frac{w(x+y)}{w(x) w(y)} d x d y \\
& \geq \int_{\left(E^{+} \cap \mathbb{R}_{+}^{n}\right) \times\left(E^{+} \cap \mathbb{R}_{+}^{n}\right)} \chi_{G}^{++}(|x|+|y|) B(|x|,|y|) d x d y \\
& \geq c \int_{E^{+}} \chi_{G}^{++}(r) W(r) d r_{1} \ldots d r_{n},
\end{aligned}
$$

we get $I$ dominated by the right side of (27).

Using the notations $\mathcal{G}$ and $\mathcal{G}_{x}$ as in case 2 above, term $I I$ in (31) is seen to be at most

(35) $\left(\underset{\widetilde{E} \times \widetilde{E}^{\mathrm{c}}}{\int}+\underset{\widetilde{E}^{\mathrm{c}} \times \widetilde{E}}{\int}+\iint_{\widetilde{E}^{\mathrm{c}} \times \widetilde{E}^{\mathrm{c}}} \int_{\mathcal{G}_{x}}(y) B(|x|,|y|) d x d y=I I_{1}+I I_{2}+I I_{3}\right.$. 
Now,

$\begin{aligned} I I_{1} & =\int_{\widetilde{E}}\left\{\int_{\widetilde{E}^{\mathrm{c}}} \chi_{\mathcal{G}_{x}}(y) B(|x|,|y|) d y\right\} d x \\ & \leq C \int_{E^{+}}\left\{\frac{\left|\mathcal{G}_{x}\right|}{|E|} \int_{E^{+}} B(|x|,|y|) d y\right\} d x \quad \text { by }(21) \\ & \leq C \iint_{E^{+}} \int_{\times E^{+}} \frac{|G|}{|E|} B(|x|,|y|) d x d y \quad \text { since }\left|\mathcal{G}_{x}\right| \leq 2^{n}|G| .\end{aligned}$

Similarly,

$$
I I_{2} \leq C \underset{E^{+} \times E^{+}}{\int} \int_{|E|} \frac{|G|}{|E|} B(|x|,|y|) d x d y
$$

Again,

$$
\begin{aligned}
I I_{3} & \leq C|E \| G| B\left(r_{\widetilde{E}}, r_{\widetilde{E}}\right) \quad \text { by Lemma 11(i) } \\
& \leq C \iint_{E^{+} \times E^{+}} \frac{|G|}{|E|} B(|x|,|y|) d x d y .
\end{aligned}
$$

But the common right side of (36), (37) and (38) is no bigger than

$$
\iint_{E^{+} \times E^{+}} \chi_{G}^{++}(x+y) B(x, y) d x d y,
$$

since $\chi_{G}^{++}\left(2 r_{E^{+}}\right)=C|G| /|E|$, which is dominated, in turn, by the right side of (18).

Next, we show that when $w \in \mathcal{M}_{\infty},(28)$ and (13) are equivalent. Suppose (28) holds. Taking $E=F=B_{r}(0)$ and $G=B_{r+\delta}(0)-B_{r-\delta}(0)$, $0<\delta \leq r / 2$, in (28) yields

$$
\begin{aligned}
& \iint_{|x|<r|y|<r} \chi_{G}(x+y) \frac{w(x+y)}{w(x) w(y)} d y d x \\
& \leq C \iint_{|x|<r} \int_{|y|<r} \chi_{G}^{+}(x+y) \frac{w(x+y)}{w(x) w(y)} d y d x
\end{aligned}
$$

On the left side of (39) restrict attention to $x$ and $y$ in the first orthotant and make the substitution $t_{k}=x_{k}+y_{k}, s_{k}=x_{k}, t=\sum_{k} t_{k}, s=\sum_{k} s_{k}$ to get the lower bound 


$$
\begin{array}{rl}
C \int_{0}^{r-\delta} s^{n-1} \int_{r-\delta}^{r+\delta} B(s, t-s) t^{n-1} & d t d s \\
& \geq c \int_{0}^{r-\delta} s^{n-1} B(s, r+\delta-s) \int_{r-\delta}^{r+\delta} t^{n-1} d t d s \\
& \geq c \delta r^{n-1} \int_{0}^{r-\delta} \frac{w(r+\delta)}{w(r+\delta-s) w(s)} s^{n-1} d s .
\end{array}
$$

As for the right side of (39), with $\varepsilon^{n}=c \delta r^{n-1}$, it is dominated by

$$
C \int_{|x| \leq r} \int_{B_{\varepsilon}(-x)} \frac{w(\varepsilon)}{w(x) w(y)} d y d x<\infty,
$$

since $w \in \mathcal{M}_{\infty}$. We conclude

$$
\begin{array}{rl}
\varepsilon^{n} \int_{0}^{r / 2} \frac{w(r+\delta)}{w(r+\delta-s) w(s)} s^{n-1} & d s \\
\leq C w(\varepsilon) \int_{0}^{r} s^{n-1} \frac{d s}{w(s)} \int_{B_{\varepsilon}(-x)} \frac{d y}{w(y)}<\infty .
\end{array}
$$

Dividing by $\varepsilon^{n}$ and letting $\varepsilon \rightarrow 0+$, we obtain (13).

Now suppose that $w \in \mathcal{M}_{\infty}$ and that (13) holds. With a view to bounding $I$ in (31) by the right side of (28) we claim that, given (13),

$$
\int_{0}^{r_{\tilde{E}}} \frac{w(r)}{w(|r-s|) w(s)} s^{n-1} d s \leq C \int_{0}^{r_{E^{+}}} s^{n-1} \frac{d s}{w(s)^{2}}, \quad 0 \leq r \leq r_{E^{+}} .
$$

For $r_{\widetilde{E}} \leq r \leq r_{E^{+}}$, the left side of (40) is at most

$$
\begin{gathered}
\int_{0}^{r_{\tilde{E}}} \frac{w\left(r_{\widetilde{E}}\right)}{w\left(r_{\widetilde{E}}-s\right) w(s)} s^{n-1} d s \quad \text { since } w \in \mathcal{M} \\
\leq C \int_{0}^{r_{E}+} s^{n-1} \frac{d s}{w(s)^{2}} \quad \text { by }(13) .
\end{gathered}
$$

When $0 \leq r \leq r_{\widetilde{E}}$,

$$
\int_{0}^{r} \frac{w(r)}{w(r-s) w(s)} s^{n-1} d s \leq C \int_{0}^{r_{E+}} \frac{s^{n-1}}{w(s)^{2}} d s
$$


by (13), while, arguing as for (33),

$$
\begin{aligned}
\int_{r}^{r_{\tilde{E}}} \frac{w(r)}{w(s-r) w(s)} s^{n-1} d s & =\int_{0}^{r_{\tilde{E}}-r} \frac{w(r)}{w(r+s) w(s)}(r+s)^{n-1} d s \\
& \leq C \int_{0}^{r_{\tilde{E}}} \frac{w\left(r_{\widetilde{E}}\right)}{w\left(r_{\widetilde{E}}-s\right) w(s)} s^{n-1} d s \leq C \int_{0}^{r_{E}} \frac{s^{n-1}}{w(s)^{2}} d s
\end{aligned}
$$

by (13). This proves (40), so we have

$$
\text { (41) } I \leq C \int_{E^{+}} \chi_{G}\left(r_{1}, \ldots, r_{n}\right) d r_{1} \ldots d r_{n} \int_{0}^{r_{E^{+}}} \frac{s^{n-1}}{w(s)^{2}} d s \leq C|G| \int_{E^{+}} \frac{d x}{w(x)^{2}} \text {. }
$$

We now show (41) holds with $I$ replaced by $I I$. By symmetry, $I I_{1}$ in (35) satisfies

$$
\begin{aligned}
I_{1} & \leq C \underset{\widetilde{E} \cap \mathbb{R}_{+}^{n}}{\int}\left\{\int_{\left(E^{+} \cap \mathbb{R}_{+}^{n}\right)^{c}} \chi_{\mathcal{G}_{x}}(y) B(|x|,|y|) d y\right\} d x \\
& \leq C \underset{\widetilde{E} \cap \mathbb{R}_{+}^{n}}{\int}|G| B\left(|x|, r_{\widetilde{E}}-|x|\right) d x=C|G| \int_{0}^{r_{\tilde{E}}} B\left(r_{\widetilde{E}}-s, s\right) s^{n-1} d s \\
& \leq C|G| \int_{0}^{r_{E}} \frac{s^{n-1}}{w(s)^{2}} d s,
\end{aligned}
$$

by (13). The term $\mathrm{II}_{2}$ in (35) is dealt with similarly. Again,

$$
\begin{aligned}
I_{3} & \leq C|E||G| B\left(r_{\widetilde{E}}, r_{\widetilde{E}}\right) \\
& \leq C|G| \int_{0}^{r_{\tilde{E}}} B\left(r_{\widetilde{E}}-s, s\right) s^{n-1} d s \leq C|G| \int_{0}^{r_{E^{+}}} \frac{s^{n-1}}{w(s)^{2}} d s
\end{aligned}
$$

by (13). Since $w \in \mathcal{M}_{\infty}$,

$$
\frac{|G|}{w(x)^{2}} \leq C \int_{-G^{+} \cap \mathbb{R}_{+}^{n}} \frac{w(-y)}{w(x-y) w(x)} d y,
$$

whence, by (41) (for $I I$ as well as for $I$ ), the left side of (28) is at most

$$
\begin{aligned}
\int_{E^{+} \cap \mathbb{R}_{+}^{n}-G^{+} \cap \mathbb{R}_{+}^{n}} \frac{w(-y)}{w(x-y) w(x)} d y d x & \leq C \int_{E^{+} \cap \mathbb{R}_{+}^{n}\left(-G^{+} \cap \mathbb{R}_{+}^{n}\right)-x} \frac{w(x+y)}{w(x) w(y)} d y d x \\
& \leq C \iiint_{E^{+} \times E^{+}} \chi_{G^{+}}(x+y) \frac{w(x+y)}{w(x) w(y)} d y d x
\end{aligned}
$$

which completes the proof. 
4. Necessary conditions. In this section we prove the necessity half of Theorems 1 and 2. In fact, we show that, given $w \in \mathcal{M}, X(w)$ closed under convolution implies (11). But first we prove simpler necessary conditions which are valid in a wider context than that of Theorem 1 or 2 .

LEMMA 12. Suppose $w$ is even on $\mathbb{R}^{n}$, i.e. $w(x)=w(-x)$ for all $x \in \mathbb{R}^{n}$. If $X=X\left(\mathbb{R}^{n}\right)$ is an r.i. space and $X(w)$ is closed under convolution, then $X(w) \subset L^{1}\left(\mathbb{R}^{n}\right)$ or, equivalently, $w^{-1} \in X^{\prime}$. Moreover, if $C>0$ is as in (8), then

$$
\|f\|_{L^{1}} \leq C\|f\|_{X(w)} \quad \text { for } f \in X(w) .
$$

Proof. Fix $f \in X(w)$ with $\|f\|_{X(w)}=1$ and define $T: X(w) \rightarrow X(w)$ by $(T g)(x)=(|f| * g)(x), x \in \mathbb{R}^{n}$. By $(8), T$ is bounded on $X(w)$ with norm at most $C$ and, by duality, $T^{\prime}$ is bounded on $X(w)^{\prime}=X^{\prime}\left(w^{-1}\right)$ with norm at most $C$. But, since $w$ is even, $T^{\prime}=T$, so, by Theorem 3 and Corollary $5,|f| * L^{2} \subset L^{2}$ with norm at most $C$ and it follows that

$$
\begin{aligned}
\left.\left.\int_{\mathbb{R}^{n}}|| f\right|^{\wedge}(\zeta)\right|^{2}|g(\zeta)|^{2} d \zeta & =\int_{\mathbb{R}^{n}}|(|f| * \widehat{g})(x)|^{2} d x \\
& \leq C \int_{\mathbb{R}^{n}}|\widehat{g}(x)|^{2} d x=C \int_{\mathbb{R}^{n}}|g(\zeta)|^{2} d \zeta
\end{aligned}
$$

for all $g \in L^{2}$. Thus $\left.|| f\right|^{\wedge}(\zeta) \mid$ is bounded by $C$ and, in particular,

$$
\|f\|_{1}=\int_{\mathbb{R}^{n}}|f|=|f|^{\wedge}(0) \leq C=C\|f\|_{X(w)} .
$$

Lemma 13. Suppose $w$ is radial, finite a.e. and satisfies

$$
B\left(r_{1}, s\right) \leq C B\left(r_{2}, s\right) \quad \text { for } s>0, r_{1} \geq r_{2}>0,
$$

yet fails to satisfy

$$
w(y) \leq C w(z) \quad \text { for } 0<y<z
$$

for the same constant $C$; that is,

$$
w(y)>C w(z) \quad \text { for some } 0<y<z .
$$

Then $\left(w^{-1}\right)^{+}(x)=\infty$ for all $x \in \mathbb{R}^{n}$.

Proof. $w$ radial and finite a.e. implies there exists $M>0$ and a set $E \subset\left\{x \in \mathbb{R}_{+}^{n}: y \leq|x| \leq z\right\},|E|>0$, with $w(x) \leq M$ for all $x$ with $|x| \in E$. We will be done if we can show that for each $k=1,2, \ldots$,

$$
w(x) \leq M r^{k} \quad \text { for }|x| \in E+k(z-y),
$$

where $r=C w(z) / w(y)<1$ by (44). But, for $|x| \in E+k(z-y)$, say $|x|=u+k(z-y), u \in E$, we have 


$$
\begin{aligned}
w(x)=w(|x|) & =w(u) \prod_{j=0}^{k-1} \frac{w(u+(j+1)(z-y))}{w(u+j(z-y))} \\
& \leq M \prod_{j=0}^{k-1} C \frac{w(z)}{w(y)} \quad \text { by }(42) \\
& \leq M r^{k} .
\end{aligned}
$$

Corollary 14. If $w$ is radial and satisfies (42) and $X(w)$ is closed under convolution, then (43) holds.

Proof. By Lemma $12, w^{-1} \in X^{\prime}$, which means $\left(w^{-1}\right)^{+}(x)<\infty$ for all $0 \neq x \in \mathbb{R}^{n}$. We suppose now that $w$ satisfies $(42), X=X\left(\mathbb{R}^{n}\right)$ is an r.i. space and $X(w)$ is closed under convolution (i.e. (8) holds) and prove that (11) holds. Begin by fixing $r>0$. For $g(x)=g(|x|) \geq 0$ we have

$$
\begin{aligned}
& \int_{|x|<r}\left(\left(\chi_{B_{r}(0)} w^{-1}\right) *\left(g w^{-1}\right)\right)(x) w(x) d x \\
& \quad \leq\left\|\left(\chi_{B_{r}(0)} w^{-1}\right) *\left(g w^{-1}\right)\right\|_{X(w)}\left\|\chi_{B_{r}(0)}\right\|_{X^{\prime}} \\
& \quad \leq C\left\|g w^{-1}\right\|_{X(w)}\left\|\chi_{B_{r}(0)} w^{-1}\right\|_{X(w)}\left\|\chi_{B_{r}(0)}\right\|_{X^{\prime}} \quad \text { by (8) } \\
& \quad \leq C\left\|\chi_{B_{r}(0)}\right\|_{X}\left\|\chi_{B_{r}(0)}\right\|_{X^{\prime}}\|g\|_{X} \leq C r^{n}\|g\|_{X}
\end{aligned}
$$

by (15). Now, the left side of (45) is

$$
\text { (46) } \begin{aligned}
\int_{|x|<r} \int_{|y|<r} & \frac{w(x)}{w(x-y) w(y)} g(y) d y d x \\
& \geq c r^{n} \int_{|y|<r / 2} g(y) \frac{1}{r^{n}-|y|^{n}} \int_{|y| \leq|x| \leq r} \frac{w(x)}{w(x-y) w(y)} d x d y \\
& \geq c r^{n} \int_{|y|<r / 2} g(y) \frac{1}{r^{n}-|y|^{n}} \int_{\substack{|y| \leq|x| \leq r \\
x-y \in \mathbb{R}_{+}^{n}}} \frac{w(x)}{w(x-y) w(y)} d x d y \\
& \geq c r^{n} \int_{|y|<r / 2} g(y) \frac{1}{r^{n}-|y|^{n}} \int_{|y|}^{r} B(s-|y|,|y|) s^{n-1} d s d y \\
& \geq c r^{n} \int_{|y|<r / 2} g(y) B(r-|y|,|y|) d y \text { since } w \in \mathcal{M} \\
& \geq c r^{n} \int_{\mathbb{R}^{n}} g(y) \chi_{B_{r}^{n}}^{n}(0)(y) B(r-|y|,|y|) d y .
\end{aligned}
$$


Combining (45) and (46) yields

$$
\int_{\mathbb{R}^{n}} \chi_{B_{r / 2}(0)}(y) B(r-|y|,|y|) g(y) d y \leq C\|g\|_{X},
$$

which, by duality, implies

$$
\left\|\chi_{B_{r / 2}(0)}(y) B(r-|y|,|y|)\right\|_{X^{\prime}(d y)} \leq C .
$$

Thus, given $x \in \mathbb{R}^{n}$, we have, by (43),

$$
\begin{aligned}
& \left\|\chi_{B_{|x| / 2}(0)}(y) \frac{w(x)}{w(x-y) w(y)}\right\|_{X^{\prime}(d y)} \\
& \leq C\left\|\chi_{B_{|x| / 2}(0)}(y) B(|x|-|y|,|y|)\right\|_{X^{\prime}(d y)} \leq C .
\end{aligned}
$$

From (43) and the rearrangement-invariance of $X^{\prime}$ we further obtain for all $z \in \mathbb{R}^{n},|z|=|x|$,

$$
\begin{aligned}
\left\|\chi_{B_{|x| / 2}(z)}(y) \frac{w(x)}{w(x-y) w(y)}\right\|_{X^{\prime}(d y)} & \leq\left\|\chi_{B_{|x| / 2}(x)}(y) \frac{w(x)}{w(x-y) w(y)}\right\|_{X^{\prime}(d y)} \\
& \leq\left\|\chi_{B_{|x| / 2}(0)}(y) \frac{w(x)}{w(x-y) w(y)}\right\|_{X^{\prime}(d y)} .
\end{aligned}
$$

As $B_{|x|}(0)$ is covered by $B_{|x| / 2}(0)$, together with a finite number (independent of $x$ ) of $B_{|x| / 2}(z),|z|=|x|$, we conclude

$$
\left\|\chi_{B_{|x|}(0)}(y) \frac{w(x)}{w(x-y) w(y)}\right\|_{X^{\prime}(d y)} \leq C .
$$

By (43) again,

$$
\begin{aligned}
& \left\|\chi_{\mathbb{R}^{n}-B_{|x|}(0)}(y) \frac{w(x)}{w(x-y) w(y)}\right\|_{X^{\prime}(d y)} \\
& \leq C\left\|\chi_{\mathbb{R}^{n}-B_{|x|}(0)}(y) \frac{1}{w(x-y)}\right\|_{X^{\prime}(d y)} \leq C\left\|w^{-1}\right\|_{X^{\prime}} \leq C,
\end{aligned}
$$

in view of Lemma 12, and, together, (47) and (48) yield (11).

\section{Examples. Let}

$$
w(x)=\left\{\begin{array}{l}
1, \\
9^{k}\left[3^{k}-\left(1-3^{-k}\right)|| x\left|-2 \cdot 3^{k}\right|\right], \\
3^{k}<|x|<3
\end{array}\right.
$$

We will prove that $w$ satisfies (11) for all r.i. spaces $X$, yet $L^{p q}(w)$ is not an algebra when $1<p<q \leq \infty$.

The assertion concerning (11) is an immediate consequence of the fact that $L^{1} \cap L^{\infty}$ is the smallest r.i. space and 
Lemma 15. Let $w$ be defined on $\mathbb{R}$ by (49). Then

$$
\left\|\frac{w(x)}{w(x-y) w(y)}\right\|_{L^{1} \cap L^{\infty}(d y)} \leq C \quad \text { for a.e. } x \in \mathbb{R}^{n} .
$$

Proof. It is sufficient to consider $x>0$, indeed $x>3$. Let $j$ and $k$ be integers, $k \geq 1$ and $0 \leq j \leq k-1$, such that

$$
3^{k}-3^{j+1}<\left|x-2 \cdot 3^{k}\right|<3^{k}-3^{j}+1 \text {. }
$$

We show

$$
\frac{w(x)}{w(x-y) w(y)} \leq 324\left(\frac{1}{w(x-y)}+\sum_{i=-1}^{1} W_{k+i}(y)\right)
$$

where

$$
W_{l}(y)=\frac{1+9^{l} H\left(|y|-3^{l}\right)}{w(y)}, \quad l=0,1,2, \ldots
$$

( $H=\chi_{\mathbb{R}_{+}}$being the Heaviside function) is readily seen to be in $L^{1} \cap L^{\infty}(d y)$ uniformly in $l$. Observe that $w(x) \leq 4 \cdot 9^{k+j+1}$ and consider the following cases for $y$, assuming $j \geq 1$.

Case $1:\left|y-2 \cdot 3^{k}\right|<3^{k}-3^{j-1}$. Here, $w(y) \geq 9^{k+j-1}$, so $w(x) / w(y) \leq$ 324 and

$$
\frac{w(x)}{w(x-y) w(y)} \leq \frac{324}{w(x-y)} .
$$

Case 2: $3^{k}-3^{j-1}<\left|y-2 \cdot 3^{k}\right|<3^{k}+2 \cdot 3^{k-1}$. We have $y>3^{k-1}$ and

$$
|x-y| \geq\left|y-2 \cdot 3^{k}\right|-\left|x-2 \cdot 3^{k}\right|>3^{j}-3^{j-1}-1 \geq 3^{j-1},
$$

so $w(x-y) \geq 9^{j-1}$ and

$$
\frac{w(x)}{w(x-y) w(y)} \leq \frac{4 \cdot 9^{k+j+1}}{9^{j-1} w(y)} \leq 324 W_{k-1}(y) .
$$

Case $3:\left|y-2 \cdot 3^{k}\right|>3^{k}+2 \cdot 3^{k-1}, y>0$. Either $0<y<3^{k-1} \leq x / 2$ and we are done by symmetry, or $y \geq 3^{k+1}+2 \cdot 3^{k-1}$, which means $y-x \geq 3^{k-1}$, $w(x-y) \geq 9^{k-1}$ and

$$
\frac{w(x)}{w(x-y) w(y)} \leq 4 W_{k+1}(y) .
$$

Case $4: y<0$. If $-3^{j-1}<y<0$, then $3^{k}<x-y<3^{k+1}$ and

$$
\left|x-y-2 \cdot 3^{k}\right| \leq\left|x-2 \cdot 3^{k}\right|+|y|<3^{k}-3^{j}+3^{j-1}+1,
$$

so $w(x-y) \geq 9^{k+j-1}, w(x) / w(x-y) \leq 324$, whence

$$
\frac{w(x)}{w(x-y) w(y)} \leq \frac{324}{w(y)} .
$$


If $y<-3^{j-1}$, then $w(y) \geq 9^{j-1}$, and

$$
\frac{w(x)}{w(x-y) w(y)} \leq 324 W_{k}(y) .
$$

Finally, when $j=0$, one of $y$ and $x-y$ is greater than $3^{j-1}$. Therefore, (50) holds then also.

To see that $L^{p q}(w)$ is not an algebra when $1<p<q \leq \infty$, let $N$ be a large positive integer and set $f=\sum_{k=1}^{n} 3^{-k} \chi_{E_{k}}$, where $E_{k}=\bigcup I_{j}$ and $I_{j}=\left(3^{j}, 3^{j}+3 / 2\right)$ for $3^{k p+1} \leq j \leq 3^{(k+1) p}$. We show that

$$
\left\|w\left(\frac{f}{w} * \frac{f}{w}\right)\right\|_{L^{p q}} \leq C\|f\|_{L^{p q}}^{2}
$$

implies

$$
N^{1 / p+1 / q} \leq C N^{2 / q}
$$

with $C>0$ independent of $N$, and hence that $q \leq p$.

Since $\mu_{f}(t) \leq\left(3^{p+1} / 2\right) t^{-p} \chi_{\left(3^{-N}, 3^{-1}\right)}(t)$, we have

$$
\|f\|_{L^{p q}}^{2} \leq 3^{2(p+1)} N^{2 / q} .
$$

Next, $(f * f)(x) \neq 0$ only when $(f * f)(x)=\int_{I_{j}} f(x-y) f(y) d y$ and $x \in I_{j}+I_{j^{\prime}}$ for some $j$ and $j^{\prime}$; moreover, for $x \in I_{j}+I_{j}^{\prime}$ and $y \in I_{j}$,

$$
\frac{w(x)}{w(x-y) w(y)} \geq \frac{1}{1000} \text {. }
$$

Thus,

$$
\begin{aligned}
w(x)\left(\frac{f}{w} * \frac{f}{w}\right)(x) & =\int_{\mathbb{R}} \frac{w(x)}{w(x-y) w(y)} f(x-y) f(y) d y \\
& \geq \frac{1}{1000}(f * f)(x)=\frac{1}{1000} \sum_{k=1}^{n} 3^{-k} \int_{E_{k}} f(x-y) d y .
\end{aligned}
$$

Suppose, now, that $3^{-N}<t \leq 3^{-1}$ and that the positive integer $l$ satisfies $3^{-l-1}<t \leq 3^{-l}$. Then

$$
\begin{aligned}
\left|\left\{x: w(x)\left(\frac{f}{w} * \frac{f}{w}\right)(x)>\frac{t}{1000}\right\}\right| & \geq\left|\left\{x:(f * f)(x)>3^{-l}\right\}\right| \\
& \geq \sum_{k=1}^{l}\left|\left\{x: \int_{E_{k}} f(x-y) d y>3^{-(l-k)}\right\}\right| \\
& \geq \sum_{k=1}^{l} 3^{k p} 3^{(l-k) p}=l 3^{l p} \geq \frac{1}{\log 3} \frac{\log \frac{1}{3 t}}{(3 t)^{p}} .
\end{aligned}
$$


It follows that

$$
\left\|w\left(\frac{f}{w} * \frac{f}{w}\right)\right\|_{L^{p q}} \geq C N^{1 / p+1 / q}>0,
$$

$c>0$ independent of $N$, and so we are done.

In the case $p>q$ we are unable to construct a weight $w$ satisfying

$$
\left\|\frac{w(x)}{w(x-y) w(y)}\right\|_{L^{p^{\prime} q^{\prime}}(d y)} \leq C, \quad x \in \mathbb{R}^{n}
$$

$\left(\left(L^{p q}\right)^{\prime}=L^{p^{\prime} q^{\prime}}\right)$ for which $L^{p q}(w), p>q$, is not an algebra, though we believe such a $w$ exists. In any event, we can show Nikol'skiı's proof will not work in this case, since (9) does not hold for $X=L^{p q}$ when $p>q$. (Of course, what we just proved implies (9) does not hold for $L^{p q}$ when $q>p$.)

Indeed, as we now prove, (9) with $X=L^{p q}$ implies $p \leq q$. For, take $f=g=\chi_{E_{N}}$, where $E_{N}=\bigcup_{k=1}^{N} I_{k}$, with $I_{k}=\left[4^{k}, 4^{k}+1 / k\right], k=1, \ldots, N$. Then,

whence

$$
\left|E_{N}\right|=\sum_{k=1}^{N} \frac{1}{k} \leq C \log N
$$

We claim

$$
\left\|\chi_{E_{N}}\right\|_{L^{p q}}^{2} \leq C\left|E_{N}\right|^{2 / p} \leq C(\log N)^{2 / p}
$$

so that (9) entails $(\log N)^{1 / p+1 / q} \leq C(\log N)^{2 / p}$ and so $p \leq q$. Observe that the left side of (51) equals

$$
\left\|\left(\chi_{E_{N}} * \chi_{E_{N}}\right)^{1 / p}\right\|_{L^{p q}} \geq C\left\{\int_{N^{-2 / p}}^{N^{-1 / p}}\left|\left\{\chi_{E_{N}} * \chi_{E_{N}}>2 t^{p}\right\}\right|^{q / p} t^{q-1} d t\right\}^{1 / q} .
$$

Now,

$$
\chi_{E_{N}} * \chi_{E_{N}} \geq 2 \sum_{j=1}^{N} \sum_{k=j}^{N} \frac{1}{k} \chi_{I_{j, k}},
$$

where the $I_{j, k}=\left[4^{j}+4^{k}+1 / k, 4^{j}+4^{k}+1 / j\right]$ are pairwise disjoint. So, when $N^{-2 / p}<t<N^{-1 / p}$,

$$
\begin{aligned}
\left|\left\{\chi_{E_{N}} * \chi_{E_{N}}>2 t^{p}\right\}\right| & \geq \sum_{j=1}^{T} \sum_{k=j}^{T}\left(\frac{1}{j}-\frac{1}{k}\right), \quad T=\left[\frac{1}{t^{p}}\right] \\
& \geq \frac{1}{2} \sum_{j=1}^{[T / 3]} \sum_{k=2 j}^{T} \frac{1}{j} \geq \frac{1}{2}(T-2[T / 3]) \sum_{j=1}^{[T / 3]} \frac{1}{j} \geq c \frac{\log N}{t^{p}} .
\end{aligned}
$$


Thus,

$$
\begin{aligned}
\left\|\left(\chi_{E_{N}} * \chi_{E_{N}}\right)^{1 / p}\right\|_{L^{p q}} & \geq c\left\{\int_{N^{-2 / p}}^{N^{-1 / p}}\left(\frac{\log N}{t^{p}}\right)^{q / p} t^{q-1} d t\right\}^{1 / q} \\
& \geq c(\log N)^{1 / p}\left\{\int_{N^{-2 / p}}^{N^{-1 / p}} \frac{d t}{t}\right\}^{1 / q} \geq c(\log N)^{1 / p+1 / q} .
\end{aligned}
$$

Acknowledgements. We would like to thank M. Cwikel and N. Kalton for pointing out that Theorem 1 in [6] would lead to a simplified proof of Lozanovskiı's result (Theorem 4).

\section{References}

[1] C. Bennett and R. Sharpley, Interpolation of Operators, Academic Press, 1988.

[2] A. Beurling, Sur les intégrales de Fourier absolument convergentes et leur application à une transformation fonctionnelle, Neuvième Congrès Math. Scand., Helsingfors, 1938, 345-366.

[3] D. Boyd, The Hilbert transform on rearrangement-invariant spaces, Canad. J. Math. 19 (1967), 599-616.

[4] A. P. Calderón, Intermediate spaces and interpolation, the complex method, Studia Math. 24 (1964), 113-190.

[5] M. Cwikel and P. Nilsson, Interpolation of weighted Banach lattices, Mem. Amer. Math. Soc., to appear.

[6] T. A. Gillespie, Factorization in Banach function spaces, Nederl. Akad. Wetensch. Proc. Ser. A 84 (1981), 287-300.

[7] S. Grabiner, Weighted convolution algebras on the half line, J. Math. Anal. Appl. 83 (1981), 531-583.

[8] E. Hewitt and K. A. Ross, Abstract Harmonic Analysis, II, Springer, Berlin, 1963.

[9] R. How ard and A. Schep, Norms of positive operators on $L^{p}$-spaces, Proc. Amer. Math. Soc. 109 (1990), 135-146.

[10] E. Kerlin and A. Lambert, Strictly cyclic shifts on $l_{p}$, Acta Sci. Math. (Szeged) 35 (1973), 87-94.

[11] G. Ya. Lozanovskiur, On some Banach lattices, Sibirsk. Mat. Zh. 10 (1969), 419431 (in Russian).

[12] N. K. Nikol's ki , Invariant subspaces of the shift operator in some sequence spaces, Candidate's Dissertation, Leningrad, 1966 (in Russian).

[13] F. Riesz, Sur une inégalité intégrale, J. London Math. Soc. 5 (1930), 162-168.

[14] S. L. Sobolev, On a theorem of functional analysis, Mat. Sb. 46 (1938), 471-497 (in Russian).

DEPARTMENT OF MATHEMATICS DEPARTMENT OF MATHEMATICS AND STATISTICS BROCK UNIVERSITY ST. CATHARINES, ONTARIO MCMASTER UNIVERSITY CANADA L2S 3A1 HAMILTON, ONTARIO CANADA L8S $4 \mathrm{~K} 1$ 